\title{
WEIGHTED ENTIRE FUNCTIONS
}

\author{
BY K. RAMAN UNNI ${ }^{1}$
}

Communicated by R. Boas, November 9, 1964

The aim of this note is to announce some results concerning the weighted entire function $z^{\alpha} f(z)$, where $\alpha \geqq 0$ and $f(z)$ is an entire function of exponential type. All these results are known to be true when $\alpha=0$. Boas [2] proved that if $f(z)$ is an entire function of exponential type such that $f(x) \in L^{p}(-\infty, \infty)$, then $f^{\prime}(x) \in L^{p}(-\infty, \infty)$. Plancherel and Pólya [6] showed that if $f(z)$ is an entire function of exponential type such that $f(x) \in L^{p}(-\infty, \infty)$, then $\sum_{n=-\infty}^{\infty}|f(n)|^{p}$ $<\infty$; if the type of $f(z)$ is less than $\pi$, then the converse is also true. Harvey [4] proved a number of results concerning the pth mean values of an entire function of exponential type. In this note we generalize all these results to weighted entire functions. These results will appear with proofs soon.

Let $z=x+i y$, where $x$ and $y$ are real, denote the complex variable. Suppose $p>0, \alpha \geqq 0$ and $f(z)$ is an entire function of exponential type. We set, for each fixed real number $a$,

$$
M_{T}^{p, \alpha}[f(x+a+i y)]=\frac{1}{2 T} \int_{-T}^{T}\left|x^{\alpha} f(x+a+i y)\right|^{p} d x .
$$

If $n$ is a positive integer, we set, for each fixed integer $m$,

$$
N_{n}^{p, \alpha}[f(x+m)]=(2 n+1)^{-1} \sum_{k=-n}^{n}\left|k^{\alpha} f(k+m)\right|^{p} .
$$

We define

$$
M^{p, \alpha}[f(x+i y)]=\limsup _{T \rightarrow \infty} M_{T}^{p, \alpha}[f(x+i y)]
$$

and

$$
N^{p, \alpha}[f(x)]=\limsup _{n \rightarrow \infty} N_{n}^{p, \alpha}[f(x)] .
$$

Here $M^{p, \alpha}[f(x)]$ and $M^{p, \alpha}[f(x+i y)]$ are the weighted $p$ th mean of $f(z)$ along the real axis and along a line parallel to the real axis, re-

${ }^{1}$ Presented to the Society, April 30, 1963. The contents of this paper form part of the author's dissertation at Northwestern University (August, 1963), written under the direction of Professor R. P. Boas, Jr. This research was partially supported by NSF Grant GP-314. 
spectively, with weight $x^{\alpha}$, whereas $N^{p, \alpha}[f(x)]$ is the weighted mean at the integers.

Our principal result is

Theorem. Let $\alpha>0, p>0$ and let $f(z)$ be an entire function of exponential type $\tau$. Then

(1) $x^{\alpha} f(x) \in L^{p}(-\infty, \infty)$ implies $\sum_{n=-\infty}^{\infty}\left|n^{\alpha} f(n)\right|^{p}<\infty$;

(2) $x^{\alpha} f(x) \in L^{p}(-\infty, \infty)$ implies

$$
\int_{-\infty}^{\infty}\left|x^{\alpha} f(x+i y)\right|^{p} d x \leqq e^{p \tau|y|} \int_{-\infty}^{\infty}\left|x^{\alpha} f(x)\right|^{p} d x
$$

(3) $x^{\alpha} f(x) \in L^{p}(-\infty, \infty)$ implies $x^{\alpha} f^{\prime}(x) \in L^{p}(-\infty, \infty)$;

(4) $M^{p, \alpha}[f(x+a+i y)]=M^{p, \alpha}[f(x+i y)]$ for each fixed real number $a$;

(5) $N^{p, \alpha}[f(x+m)]=N^{p, \alpha}[f(x)]$ for each fixed integer $m$;

(6) if $M^{p, \alpha}[f(x)]=A<\infty$, then

$$
x^{\alpha} f(x)=O\left(|x|^{1 / p}\right) \text { as }|x| \rightarrow \infty ;
$$

(7) if $N^{p, \alpha}[f(x)]=A<\infty$, and $n$ is an integer, then

$$
n^{\alpha} f(n)=O\left(|n|^{1 / p}\right) \quad \text { as } \quad|n| \rightarrow \infty ;
$$

(8) $M^{p, \alpha}[f(x+i y)] \leqq e^{p r|y|} M^{p, \alpha}[f(x)]$;

$$
M^{p, \alpha}\left[f^{\prime}(x)\right] \leqq \frac{(p+2) 2^{p+2}}{p \pi \tau \delta^{p+1}}\left(e^{p \tau \delta}-1\right) M^{p, \alpha}[f(x)],
$$

where $\delta$ is an arbitrary positive number;

(10) $M^{p, \alpha}[f(x)]<\infty$ implies $M^{p, \alpha}[f(x+i y)]$ is a continuous function of $y$ provided $p>1$; that

(11) there exists a constant $B>0$ which depends on $p$ and $\tau$ only such

$$
N^{p, \alpha}[f(x)] \leqq B M^{p, \alpha}[f(x)]
$$

Moreover, if $\tau<\pi$,

(12) converse of (1) is true;

(13) converse of (11) is true; more precisely, there exists a constant $C$ which depends on $p, \tau$ and $\alpha$ such that

$$
M^{p, \alpha}[f(x)] \leqq C N^{p, \alpha}[f(x)] .
$$

The results (4)-(11) and (13) are generalizations of those of Harvey [4] and the methods and techniques are analogous to his.

Note. (1) and (12) can be easily proved by the interpolation formulae and the techniques used in the proof of (11) and (13). These 
results are also contained in a theorem of Korevaar [5]. If the type of $f(z)$ is $\pi$, that the conclusions of (12) and (13) may not be true can easily be seen by considering the case when $f(z)=\sin \pi z$ and $\alpha=1$.

\section{REFERENCES}

1. R. P. Boas, Jr., Entire functions, Academic Press, New York, 1954.

2. - Representations for entire functions of exponential type, Ann. of Math. (2) 39 (1938), 269-286.

3. G. H. Hardy, J. E. Littlewood and G. Pólya, Inequalities, Cambridge Univ. Press, New York, 1934.

4. A. R. Harvey, The mean of a function of exponential type, Amer. J. Math. 70 (1948), 181-201.

5. J. Korevaar, Fourier transforms of generalized functions, Sympos. Harmonic Analysis and Related Integral Transforms, Vol. 2, Cornell Univ., Ithaca, N. Y., 1956.

6. M. Plancherel and G. Pólya, Fonctions entières et intégrales de Fourier multiples, Comment. Math. Helv. 9 (1938), 110-163.

NORTHWESTERN UNIVERSITY 